\title{
Efecto socioeconómico sobre las características fanerópticas de gallinas autóctonas de Ecuador
}

Toalombo, P.A. ${ }^{1,2 @ ; ~ C a m a c h o, ~ C . A . ~}{ }^{\text {; }}$ Buenaño, R. ${ }^{\text {; }}$ Jiménez, S. ${ }^{\text {; }}$ Navas-González, F.J. ${ }^{2}$; Landi, V. ${ }^{3}$ y Delgado, J.V. ${ }^{2}$

'Escuela Superior de Chimborazo, Carrera de Zootecnia, Riobamba. Ecuador.

${ }^{2}$ Departamento de Genética. Universidad de Córdoba. España.

${ }^{3}$ Animal Breeding Consulting, S.L., Córdoba. España.

Palabras ClaVe adicIONALES

Gallinas de campo.

Recursos genéticos locales.

Características fanerópticas.

Producción sustentable.

\section{RESUMEN}

\begin{abstract}
Se caracterizaron fanerópticamente 244 aves: 207 gallinas $84,84 \%$ y 37 gallos $15,16 \%$ en 25 localidades de 6 provincias del Ecuador, Región Sierra: Bolívar 31, Chimborazo 70, Tungurahua 35, Cotopaxi 32; Región Costa: Guayas 28; Región Amazónica: Morona Santiago 38. Mediante observación directa, los datos recogidos fueron tabulados en el programa estadístico SPSS versión Statistic 19, como estadísticos descriptivos se obtuvo frecuencias; además se realizó, la prueba Chi-cuadrado de Pearson y La V de Cramer $(\mathrm{P} \leq 0,05)$. La provincia de Bolívar parroquia Chimbo, obtuvo los mayores valores de la mediana para color de plumas, tipo de cresta, color de piel y presencia de plumas en el cuello 6,50; 1,00; 1,00; 1,00 respectivamente. La coloración del plumaje que se presentó fue marrón café $17,21 \%$, seguido del jaspeado $16,80 \%$, negro $14,34 \%$ y barrado $10,25 \%$. La forma más frecuente de las crestas fue la sencilla con $76,74 \%$, seguida de tipo nuez $8,20 \%$. La piel de las aves presentó una coloración amarilla en la mayoría de los casos $52,46 \%$. El mayor porcentaje de los animales presenta emplumado de tipo normal 94,26 $\%$ y $5,74 \%$ cuello desnudo. Las aves estudiadas son poblaciones heterogéneas con gran variabilidad en cuanto a características fanerópticas, que permitiría la creación de líneas productivas que conserven su fenotipo y rusticidad, tomando en consideración la preferencia de los consumidores y productores que ejecuten bajo sistemas de producción tradicionales, culturales, sociales y económicamente sustentables en beneficio de las familias campesinas salvaguardando la soberanía alimentaria y los saberes ancestrales.
\end{abstract}

\section{Socioeconomic effect on morphological traits of Ecuador autochthonous hens}

\section{SUMMARY}

\section{ADDITIONAL KEYWORDS}

Creole chickens.

Local genetic resources.

Morphological traits.

Sustainable production.

\section{INFORMATION}

\section{Cronología del artículo.}

Recibido/Received: 03.02.2019

Aceptado/Accepted: 08.09.2019

On-line: 15.07 .2019

Correspondencia a los autores/Contact e-mail:

ptoalombo@espoch.edu.ec
244 birds were characterized phaneroptically: 207 chickens $84.84 \%$ and 37 chickens $15.16 \%$ in 25 localities of 6 provinces of Ecuador, Sierra Region: Bolívar 31, Chimborazo 70, Tungurahua 35, Cotopaxi 32; Coast Region: Guayas 28; Amazon Region: Morona Santiago 38. Through direct observation, the collected data were tabulated in the statistical program SPSS Statistic version 19, as descriptive statistics, frequencies were obtained; The Chi-square test of Pearson and La V de Cramer (P $\leq 0.05$ ) were also performed. The province of Bolivar, Chimbo parish, obtained the highest values of the median for feather color, ridge type, skin color and presence of feathers in the neck 6,$50 ; 1.00 ; 1.00$; 1.00 respectively. The coloration of the plumage presented was brown coffee $17.21 \%$, followed by mottled $16.80 \%$, black $14.34 \%$ and barred $10.25 \%$. The most frequent form of the crests was the simple one with $76.74 \%$, followed by walnut type $8.20 \%$. The skin of the birds showed a yellow coloration in most cases $52.46 \%$. The greater percentage of the animals presents feathered of normal type $94,26 \%$ and $5,74 \%$ bare neck. The birds studied are heterogeneous populations with great variability in terms of phaneroptic characteristics, which would allow the creation of production lines that preserve their phenotype and rusticity, taking into account the preference of consumers and producers who run under traditional, cultural, social production systems and economically sustainable for the benefit of peasant families, safeguarding food sovereignty and ancestral knowledge.

\section{INTRODUCCIÓN}

Los recursos genéticos animales para la alimentación y la agricultura a nivel mundial, son de suma importancia (FAO (2007)). Las aves de corral, como otros animales domésticos, desempeñan funciones importantes para la subsistencia de la sociedad humana, ya que aportan con el $80 \%$ de la producción de huevos y carne (Besbes 2009), siendo importante señalar que son la base económica y cultural del sector campesino (Zaragoza et al. 2013, Tadelle, Alemu \& Peters 2000). El incremento de la avicultura intensiva sobre la avicultura de traspatio, ha causado preocupación referente a la conservación de Recursos Zoogenéticos y de manera 
directa sobre la soberanía alimentaria; por lo que su situación debido a la falta de caracterización cuantitativa y cualitativa sigue siendo delicada; así como también el seguimiento que se debe llevar a cabo a lo largo del tiempo, para verificar si las características morfológicas han variado en las diferentes regiones de Ecuador y fijarlos en los sistemas de producción del país. Al igual que de manera mundial, en Sudamérica también existe una tendencia preponderante sobre la explotación de estirpes comerciales altamente productivas, las cuales se especializan en la producción de carne y huevos. Esta situación amenaza de manera severa la extinción de las razas locales tradicionales (Moula et al. 2014), las cuales han sido la base para establecer las líneas comerciales, y con ello la pérdida de características de resistencia y adaptación al medio rural (Alemu \& Tadelle 1997; Zaragoza et al. 2013). Según (FAO 2006)) alrededor del $40 \%$ de las razas aviares no se encuentran inventariadas (Besbes, O \& Zeevi 2009). Por lo que en la presente investigación se lleva a cabo la caracterización faneróptica de las gallinas de campo de Ecuador, tomando en consideración los diferentes sistemas agroecológicos en los que se producen, (Dana, N. et al. 2011), (Kingori, Wachira \& Tuitoek 2010), (Cabarles et al. 2012) de tal manera que se evalúe la situación de los recursos locales que no se encuentran identificados y que pueden ser económicamente rentables.

\section{MATERIAL Y MÉTODOS}

Se registraron las características fanerópticas de un total de 234 aves: 207 gallinas $(84,84 \%)$ y 37 gallos (15,16\%).De la Región Sierra: Bolívar (31), Chimborazo (70), Tungurahua (35), Cotopaxi (32); de la Región Costa: Guayas (28); y de la Región Amazónica: Morona Santiago (38). Estas Regiones representan las diferentes zonas agroecológicas de Ecuador, es importante mencionar que las 25 localidades consideradas fueron tomadas en cuenta según el último Censo agropecuario 2010. Las aves estudiadas pertenecían a 50 productores que se dedican a la actividad avícola de traspatio, es decir, para autoconsumo y venta local. Se consideró que no exista relación genética entre productores. Las características fanerópticas se obtuvieron mediante observación directa y se anotaron en un fichero es- tructurado que incluyó localización georreferenciada e información del productor. Las variables cualitativas registradas para cada gallina fueron: tipo de cresta, presencia o ausencia de plumas en el cuello, color de la piel, color y tipo de plumas en el cuerpo.

Los datos se procesaron con el programa estadístico SPSS versión Statistic 19. Para determinar la dispersión de los datos se obtuvieron las frecuencias para cada variable; además se realizó la prueba Chi-cuadrado de Pearson y la V de Cramer que es una corrección aplicada al coeficiente Chi Cuadrado, lo cual permite obtener un índice con valor máximo igual a 1 (que indica la mayor asociación entre variables) o un valor mínimo de 0 (que indica NO asociación). Se consideró un valor de $\mathrm{P}<0,05$ para las diferencias significativas.

\section{RESULTADOS}

El color del plumaje, tipo de cresta, color de piel y presencia de plumas en el cuello presentan diferencias estadísticas significativas $(\mathrm{P}<0.05)$ entre provincias y parroquias. Para el color del plumaje se observa una asociación lineal entre sí alta para las provincias y media para las parroquias Tabla I. En cuanto al tipo de cresta y color de piel presentan un grado de asociación lineal medio para las provincias y alto para las parroquias. Para la variable presencia de plumas en el cuello el grado de asociación lineal entre las provincias y parroquias es medio Tabla I.

En Bolívar se obtuvo los mayores valores de la mediana para la parroquia Chimbo referente a color de plumas, tipo de cresta, color de piel y presencia de plumas en el cuello 6,50; 1,00; 1,00; 1,00 respectivamente; así también en Chimborazo la parroquia Pallatanga con 11,$00 ; 1,00 ; 3,00 ; 1,00$; en Bucay que pertenece a Guayas 7,00; 1,00; 2,00; 1,00; Tisaleo - Tungurahua 11,00; 1,00; 3,00; 1,00; Cotopaxi localidad Pujilí con 7,$00 ; 1,00 ; 1,50 ; 1,00$ y Morona Santiago - Sinaí con 10,$00 ; 1,00 ; 2,00 ; 1,00$ respectivamente, Tabla II.

Las variables fanerópticas estudiadas no presentaron diferencias significativas entre macho y hembra, por lo que las frecuencias se describen de manera general para ambos sexos. El color de plumaje es muy variado (Tabla III). El marrón café es el más común

Tabla I. Características fanerópticas en gallinas criollas del Ecuador (Morphometric characteristics in Creole chickens of Ecuador).

\begin{tabular}{|c|c|c|c|c|c|c|}
\hline & Variable & $\begin{array}{c}\text { Chi-Square Tests } \\
\text { Value }\end{array}$ & df & $\begin{array}{c}\text { Asymptotic } \\
\text { Significance } \\
\text { (2-sided) }\end{array}$ & $\begin{array}{c}\text { Cramer's V } \\
\text { Value }\end{array}$ & $\begin{array}{l}\text { Approximate } \\
\text { Significance }\end{array}$ \\
\hline \multirow[t]{4}{*}{ Provincia } & Color de plumas & $322,357^{a}$ & 90 & 0,000 & 0,514 & 0,000 \\
\hline & Tipo de cresta & $95,021^{a}$ & 15 & 0,000 & 0,360 & 0,000 \\
\hline & Color de piel & $115,133^{a}$ & 20 & 0,000 & 0,343 & 0,000 \\
\hline & $\begin{array}{l}\text { Presencia de plumas } \\
\text { en cuello }\end{array}$ & $31,345^{a}$ & 5 & 0,000 & 0,358 & 0,000 \\
\hline \multirow[t]{4}{*}{ Parroquia } & Color de plumas & $853,536^{a}$ & 432 & 0,000 & 0,441 & 0,000 \\
\hline & Tipo de cresta & $185,549^{a}$ & 72 & 0,000 & 0,503 & 0,000 \\
\hline & Color de piel & $456,763^{a}$ & 96 & 0,000 & 0,684 & 0,000 \\
\hline & $\begin{array}{l}\text { Presencia de plumas } \\
\text { en cuello }\end{array}$ & $46,924^{a}$ & 24 & 0,003 & 0,439 & 0,003 \\
\hline
\end{tabular}




\begin{tabular}{|c|c|c|c|c|c|}
\hline Provincia & Parroquia & Color de plumas & Tipo de cresta & Color de piel & $\begin{array}{l}\text { Presencia de } \\
\text { plumas en cuello }\end{array}$ \\
\hline \multirow[t]{3}{*}{ Bolívar } & Echeandía & 4,00 & 2,00 & 1,00 & 1,00 \\
\hline & San Pablo & 5,50 & 1,00 & 1,00 & 1,00 \\
\hline & Chimbo & 6,50 & 1,00 & 1,00 & 1,00 \\
\hline \multirow[t]{6}{*}{ Chimborazo } & Columbe & 6,00 & 1,00 & 1,00 & 1,00 \\
\hline & Nabuzo & 6,00 & 1,00 & 1,00 & 1,00 \\
\hline & Pallatanga & 11,00 & 1,00 & 3,00 & 1,00 \\
\hline & Licto & 10,00 & 1,00 & 2,00 & 1,00 \\
\hline & Chambo & 6,00 & 1,00 & 3,00 & 1,00 \\
\hline & Guano & 7,00 & 1,00 & 4,00 & 1,00 \\
\hline \multirow[t]{2}{*}{ Guayas } & Bucay & 7,00 & 1,00 & 2,00 & 1,00 \\
\hline & San Vicente & 3,00 & 1,00 & 3,00 & 1,00 \\
\hline \multirow[t]{5}{*}{ Tungurahua } & Pelileo & 6,50 & 1,00 & 1,00 & 1,00 \\
\hline & Tisaleo & 11,00 & 1,00 & 3,00 & 1,00 \\
\hline & Ambato & 7,00 & 1,00 & 1,00 & 1,00 \\
\hline & Baños & 7,00 & 1,00 & 3,00 & 1,00 \\
\hline & Santa Cecilia & 7,00 & 1,00 & 1,00 & 1,00 \\
\hline \multirow[t]{4}{*}{ Cotopaxi } & Pujilí & 7,00 & 1,00 & 1,50 & 1,00 \\
\hline & Poaló & 6,00 & 1,00 & 1,00 & 1,00 \\
\hline & Salcedo & 4,50 & 1,00 & 2,00 & 1,00 \\
\hline & Saquisilí & 7,00 & 1,00 & 3,00 & 1,00 \\
\hline \multirow[t]{5}{*}{ Morona Santiago } & Sevilla Don Bosco & 8,00 & 1,00 & 3,00 & 1,00 \\
\hline & Morona & 3,00 & 1,00 & 3,00 & 1,00 \\
\hline & Sinaí & 10,00 & 1,00 & 2,00 & 1,00 \\
\hline & Tres marías & 15,00 & 1,00 & 1,00 & 1,00 \\
\hline & Sevilla de Oro & 8,00 & 4,00 & 2,00 & 1,00 \\
\hline
\end{tabular}

$(17,21 \%)$, seguido del jaspeado con $(16,80 \%)$, negro con $(14,34 \%)$ y por último barrado con $(10,25 \%)$. Otros colores frecuentes que se presentan en menor cantidad son amarillo entero, colores combinados amarillo- negro- verde; café-negro, negro-amarillo, amarillo-café, blanco, marrón negro, negro rojizo, gris, multicolor, negro verdoso, pardo, blanco con gris, amarillo-gris, negro-naranja, blanco con negro, anaranjado negro. La forma más frecuente de las crestas fue la sencilla con $(76,74 \%)$, seguida de la tipo nuez $(8,20 \%)$. La piel de las aves presentó una coloración amarilla en la mayoría de los casos $(52,46 \%)$, la presencia blanca y rosada también fueron registradas con $(27,87 \%)$ y $(13,11 \%)$ respectivamente y en menor frecuencia la blanca-amarilla $(6,15 \%)$ y negra $(0,41 \%)$. El mayor porcentaje de los animales presenta emplumado de tipo normal $(94,26$ $\%)$, y $(5,74 \%)$ de cuello desnudo.

\section{DISCUSIÓN}

El color del plumaje de las gallinas criollas del Ecuador, presentan una alta variabilidad fenotípica que caracteriza a las aves locales (McAinsh et al. 2004), por la manifestación de diferentes tipos de tonalidades e innumerables combinaciones de colores que deriva de la presencia de genes multialélicos (Tadelle, D., Alemu \& Peters 2000), (Jauregui et al. 2012), con diferentes efectos e interacciones entre ellos (Periquet 1997), que las diferencian de las líneas comerciales y razas. En el presente estudio, el color marrón predomina de forma similar a lo observado con las aves de Bostwana (Badubi, Rakereng \& Marumo 2006), seguido del color negro $(14 \%)$ producto de la expresión del gen " $E$ " y sus alelos con dominancias relativas (Smith 1990), lo que difiere a lo observado por (Zaragoza et al. 2013) en poblaciones de gallinas en México que presentaron $35 \%$ plumaje negro; $35,6 \%$ jaspeado; pero coincide con las aves de Ecuador en coloraciones como el barrado y blanco; además la variedad de coloraciones se deben a la presencia de las eumelaninas responsables del color negro y azul-grisáceo, y las feomelaninas del rojo, pardo y leonado que son producto de la expresión de diversos genes (Smith 1990), (Jauregui et al. 2012), los cuales son visibles en las aves analizadas, en frecuencias relativamente bajas de 6 a 0,42\% (Crawford 1990). Es por ello que los productores prefieren gallinas de diferentes matices que pueden estar afines con una dilución entre los genes locales con los establecidos mediante razas comerciales (Juárez 1995), (Tadelle, Dessie \& Ogle 1996), (TEKETEL 1986), (Dana, Nigussie et al. 2010), (Kingori, Wachira \& Tuitoek 2010).

Las gallinas de campo en el Ecuador han pasado a ser un acervo cultural, religioso y social que juegan un 
rol importante dentro de la economía de los productores de traspatio; la demanda insatisfecha que existe en el mercado nacional de carne y huevos (proteína de alto valor biológico) con características organolépticas diferentes, al que presentan las líneas comerciales producidas bajo sistemas intensivos; brindan la pauta para solventarla, mediante la producción animal a pequeña escala, con el uso de insumos ecológicos locales. Desde el punto de vista cultural, las mejores aves son comercializadas para la alimentación en diferentes celebraciones que se ofrecen en los sectores rurales; así como los huevos de campo, llamados huevos de "gallo y gallina", son cotizados para el uso en medicina tradicional (saberes ancestrales) como para el consumo fuera y dentro de la ciudad. Por lo que la producción de aves de traspatio, es una alternativa factible para producir proteína asequible de origen animal, debido al tamaño que facilita el manejo de las aves, así como la adaptación al medio ambiente en el cual se explote, lo que da paso a una alta variabilidad genética y por ende faneróptica.

La actividad pecuaria de traspatio es ejecutada en un $90 \%$ por el matriarcado, es decir madres de familia, que a más de ejercer las gestiones del hogar "amas de casa", generan ingresos económicos emergentes, ya que cada unidad es vendida a 0,25 centavos de dólar, mientras que un huevo de gallina comercial oscila entre 0,12 a 0,15 según la oferta y demanda; las dos actividades avícolas presentan excelentes rendimientos económicos, más aun las gallinas de traspatio, cuyo

\begin{tabular}{lc}
$\begin{array}{l}\text { Tabla III. Color del plumaje de la gallina gallinas } \\
\text { criollas del Ecuador (Colour of the plumage in Creole chick- } \\
\text { ens of Ecuador). }\end{array}$ \\
\hline Pluma & Frecuencia \\
\hline Amarilla & 2.87 \\
Amarillo, Negro, Verde & 0.41 \\
Negro & 14.34 \\
Café, Negro & 6.56 \\
Negro Amarillo & 3.69 \\
Marrón Café & 17.21 \\
Jaspeado & 16.80 \\
Barrado & 10.25 \\
Amarillo Café & 0.41 \\
Blanco & 6.15 \\
Marrón Negro & 4.10 \\
Negro Rojizo & 4.51 \\
Gris & 1.23 \\
Multicolor & 0.82 \\
Negro Verdoso & 1.23 \\
Pardo & 4.10 \\
Blanco Con Gris & 1.23 \\
Amarillo Con Gris & 0.82 \\
Negro Con Naranja & 0.41 \\
Blanco Con Negro & 0.82 \\
Anaranjado Negro & 2.05 \\
\hline Total & 100 \\
\hline & \\
\hline &
\end{tabular}

sistema de producción es sostenido con especies forrajeras y alimentos alternativos y un manejo con bajo riesgo; por lo tanto desarrollan un papel fundamental en la seguridad y soberanía alimentaria del Ecuador.

Las entidades de gobierno y ONG's como parte del incentivo a la producción pecuaria, con el fin de evitar el abandono del campo, han fomentado aproximadamente hace más de 7 años, dicha actividad mediante la ejecución de programas Asociativos con productores zonales; quienes son acreedores a un número determinado de aves de líneas comerciales como la Lohmann Brown e Isa Brown (huevos), Campero INTA (Pío pío - finquero), etc; así como también razas introducidas como la Araucana y otras más, que son producidas bajo un sistema extensivo en cada hogar; todos éstos procesos se han realizado sustituyendo los genotipos avícolas locales.

El censo avícola del 2006 realizado por el MAGAP, AGROCALIDAD y CONAVE, identificó cerca de 1570 entre pequeños, medianos y grandes avicultores, sin considerar la avicultura familiar o de traspatio (El Agro 2013). Por otro lado se registraron 10 millones de gallinas en planteles avícolas durante el 2012, mientras que las criadas en campo registraron una existencia de 4,7 millones, (Censos 2012 ); lo que significa, que la información sobre la avicultura de traspatio es muy escasa, impidiendo de esta manera conocer las características y el manejo de estas aves en las distintas regiones del país (Enríquez Torres 2015).

La presencia de plumaje en el cuello que se encuentra en la mayoría de las aves muestreadas, está relacionada a la interacción genotipo $\mathrm{x}$ ambiente descrita por (Santoni et al. 2000), (Ali, Ahmed \& Aly 2003), ya que las bajas temperaturas provocarían la presencia de plumas a nivel de cuello para conservar el calor. Mientras que la ausencia de plumaje estaría asociado al gen $\mathrm{Na}$, siendo el resultado de los procesos de adaptación a pisos climáticos bajos con temperatura ambiental y humedad relativa superior, la fisiología del animal debe adaptarse, por lo que las frecuencias genéticas variarán, según el medio ambiente en el que se encuentre, como medida a mantener la temperatura corporal (termorregulación) (Hill, Hill \& McGraw 2006), (Protas \& Patel 2008), (Horst \& Mathur), tal como describe (Zaragoza et al. 2013) en las regiones altas de Chiapas de México.

En las gallinas de campo del Ecuador se observó la presencia de tipo de la cresta en su mayoría sencilla (rrpp) que coincide con los estudios realizados en la gallina de cuello desnudo en Guatemala y (Zaragoza et al. 2013) en México, así como también en las gallinas de Nigeria (Apuno, Mbap \& Ibrahim 2011). La cresta es importante, por ser una vía para la pérdida de calor en las aves (Van Kampen 1974)e>, (Ibe 1993), por lo que a mayor tamaño se garantiza un medio eficiente para la disipación del calor.

La manifestación de la piel amarilla en las aves de Ecuador se presentó en mayor frecuencia, lo que difiere con aves de Guatemala (Jauregui et al. 2012) y también con aves México (Zaragoza et al. 2013); desde el punto de vista genético el color amarillo de la piel viene determinado por el gen "piel amarilla" -w-. El 
homocigoto recesivo (ww) provoca piel amarilla, mientras que el homocigoto dominante (WW) da lugar a gallinas de piel blanca, con efectos sobre pico y tarsos (Jauregui et al. 2012). Como efecto medio ambiental la coloración de la piel puede ser producto de la alimentación suministrada (manejo), a base de pastos y forrajes principalmente de alfalfa (Medicago sativa) que contiene de 400-500 mg/kg de materia seca de xantofilas, con efecto colorante en los tejidos, piel, músculo y tarsos (RAGHAVAN 2002); también existen otros factores que pueden influir en el color de la piel como el grado de penetración de genes diferentes provenientes de aves que fueron introducidas (Ca, Aja \& Segura 2000).

\section{CONCLUSION}

Las aves de Ecuador estudiadas son poblaciones heterogéneas con gran variabilidad en características fanerópticas. Los rasgos que predominan son: el plumaje color marrón, posiblemente por la influencia de líneas comerciales; el color negro puede estar asociado a las preferencias culturales tanto de los productores como de los consumidores; además las diferentes tonalidades presentadas, son características que sirven como medio de camuflaje contra los depredadores. Se manifiesta en su mayoría cresta simple, presencia de plumaje en todo el cuerpo (termorregulación) y piel amarilla, lo que favorece el consumo de carne, ya que los consumidores relacionan calidad con color amarillo. El precio de huevo de "gallo y gallina", tiene un costo en el mercado que a pesar de ser mayor al de la gallina comercial, sigue siendo accesible y con excelente aceptación en el mercado, constituyendo una oportunidad para fomentar e incrementar la producción de traspatio y por ende favorece la conservación de los recursos genéticos, cuyos animales presentan cualidades de resistencia a condiciones ambientales, eficiencia productiva, que forman parte de la biodiversidad mundial, que hoy día se ve afectada.

\section{AGRADECIMIENTOS}

A la Escuela Superior Politécnica de Chimborazo, Carrera de Zootecnia, así como también al grupo AGR18 Departamento de Genética de la Universidad de Córdoba. Y de manera especial a todos los productores de traspatio del Ecuador, que nos permitieron realizar varias visitas a sus explotaciones.

\section{BIBLIOGRAFÍA}

Alemu, Y \& Tadelle, D 1997, 'The status of poultry research and development in Ethiopia, research bulletin No. 4, poultry commodity research program Debrezeit Agricultural research center', Alemaya University of agriculture, Ethiopia, vol. 62.

Ali, BA, Ahmed, MMM \& Aly, OM 2003, 'Relationship between genetic similarity and some productive traits in local chicken strains', African Journal of Biotechnology, vol. 2, no. 2, pp. 46-7.

Apuno, AA, Mbap, ST \& Ibrahim, T 2011, 'Characterization of local chickens (Gallus gallus domesticus) in shelleng and song local government areas of Adamawa State, Nigeria', Agriculture and Biology Journal of North America, vol. 2, no. 1, pp. 6-14.

Badubi, SS, Rakereng, M \& Marumo, M 2006, 'Morphological characteristics and feed resources available for indigenous chickens in
Botswana', Livestock Research for Rural Development, vol. 18, no. 1, pp. 205-11.

Besbes, B 2009, 'Genotype evaluation and breeding of poultry for performance under sub-optimal village conditions', World's Poultry Science Journal, vol. 65, no. 2, pp. 260-71.

Besbes, O \& Zeevi, A 2009, 'Dynamic pricing without knowing the demand function: Risk bounds and near-optimal algorithms', Operations Research, vol. 57, no. 6, pp. 1407-20.

Ca, J, Aja, M \& Segura, CJC 2000, 'Rasgos de apariencia fenotipica en la avicultura rural de los municipios de la Ribera del Lago de Patzcuaro, Michoacan, Mexico', Livestock Research for Rural Development, vol. 12, p. 1

Cabarles, JC, Lambio, AL, Vega, SA, Capitan, SS \& Mendioro, MS 2012, 'Distinct morphological features of traditional chickens (Gallus gallus domesticus L.) in Western Visayas, Philippines', Animal Genetic Resources/Resources génétiques animales/Recursos genéticos animales, vol. 51, pp. 73-87.

Censos, INDEy 2012 'Encuesta de Superficie y Producción Agropecuaria Continua'.

Crawford, RD 1990, Poultry breeding and genetics.

Dana, N, Dessie, T, van der Waaii, LH \& van Arendonk, JAM 2010, 'Morphological features of indigenous chicken populations of Ethiopia', Animal Genetic Resources/Resources génétiques animales/Recursos genéticos animales, vol. 46, pp. 11-23.

Dana, N, Megens, HJ, Crooijmans, RP, Hanotte, O, Mwacharo, J, Groenen, MA \& van Arendonk, JA 2011, 'East Asian contributions to Dutch traditional and western commercial chickens inferred from mtDNA analysis', Anim Genet, vol. 42, no. 2, pp. 125-33.

El Agro, R 2013, 'Análisis de la avicultura ecuatoriana', Revista ElAgro.

Enríquez Torres, MR 2015, 'Evaluación de dos sistemas de alimentación de tres tipos de alimentos en aves de traspatio Caupichu III, Pichincha $2015^{\prime}$

FAO 2006, 'A Strategic Approach for Conservation and Continued Use of Animal Genetic Resources', Fourth Session of ITWG for Animal GRFA, FAO, Rome.

FAOSTAT (2007), 'Statistical database. Accessible online at, FAOSTAT, no. http://faostat.fao.org.

Hill, GE, Hill, GE \& McGraw, KJ 2006, Bird coloration: mechanisms and measurements, vol. 1, Harvard University Press.

Horst, P \& Mathur, PK 'Feathering and adaptation to tropical climates', in vol. 2, pp. 79-82.

Ibe, SN 1993, 'Growth performance of normal frizzle and naked-neck chickens in a tropical environment', Nigerian Journal of Animal Production, vol. 20, no. 1, pp. 25-31.

Jauregui, R, Flores, H, Sagastume, L, Vasquez, L, Oliva, M \& Sandoval, R 2012, 'Caracterización de la Gallina del Cuello Desnudo (Gallus domesticus nudicullis), en la Región Chorti de Guatemala', Universidad de San Carlos de Guatemala.

Juárez, CA 1995, 'Producción de huevo, calidad de cascarón y balance de calcio en gallinas criollas de cuello desnudo (Na)', Rev. Los Avicultores y su Entorno. México, vol. 1, no. 1, pp. 20-2.

Kingori, AM, Wachira, AM \& Tuitoek, JK 2010, 'Indigenous chicken production in Kenya: A review', International Journal of Poultry Science, vol. 9, no. 4, pp. 309-16.

McAinsh, CV, Kusina, J, Madsen, J \& Nyoni, O 2004, 'Traditional chicken production in Zimbabwe', World's Poultry Science Journal, vol. 60, no. 2, pp. 233-46.

Moula, N, Philippe, F-X, Moussiaux, NA, Leroy, P \& Michaux, C 2014, 'Estimation of inbreeding rates and extinction risk of forty one belgian chicken breeds in 2005 and 2010', Archivos de Zootecnia, vol. 63, no. 242, pp. 389-92.

Periquet, JC 1997, 'Races de poulets, de l'élevage, produits d'hygiéne et de santé', Edition Broché.

Protas, ME \& Patel, NH 2008, 'Evolution of coloration patterns', Annual review of cell and developmental biology, vol. 24, pp. 425-46.

RAGHAVAN, V 2002,' Avicultura Profesional', vol. Volumen no. 20 págs. , pp. 14-6. 
Santoni, S, Faivre-Rampant, P, Prado, E \& Prat, D 2000, 'Ressources génétiques', Cahiers Agricultures, vol. 9, pp. 311-27.

Smith, AJ 1990, Poultry-Tropical Agriculturist series. CTA, Macmillan Publishers, London.

Tadelle, D, Alemu, Y \& Peters, KJ 2000, 'Indigenous chickens in Ethiopia: genetic potential and attempts at improvement', World's Poultry Science Journal, vol. 56, no. 1, pp. 45-54.

Tadelle, D \& Ogle, B 1996, 'Studies on scavenging poultry production system in Central Highlands of Ethiopia', MS cThesis, presented Swedish University of Agricultural Sciences, Sweden.
TEKETEL, F 1986, 'Studies on the meat production potential of some local strains of chickens in Ethiopia', Ph.D. Thesis, J.L. University of Giessen, 210 pp.

Van Kampen, M 1974, 'Physical factors affecting energy expenditure', Energy requirements of Poultry, pp. 47-59.

Zaragoza, ML, Rodríguez, H, Hernández, Z, Perezgrovas, GR, Martínez, C \& Méndez, E 2013, 'Characterization of hens Batsi Alak in the highlands of southeast Mexico', Archivos de Zootecnia, vol. 62, no. 239 , pp. 321-32. 